\title{
Enriquecimiento tecnológico y psicopedagógico del concepto de comunidades de práctica en la educación a distancia
}

\section{Technological and Psychological Enrichment of the Concept of Communities of Practice in Distance Education}

\author{
Marco Antonio González Pérez \\ Universidad Nacional Autónoma de México. México \\ marco.gonzalez@ired.unam.mx
}

\begin{abstract}
Resumen:
El presente documento aborda el tema de la transformación de las comunidades de práctica como un colectivo vigente de construcción de conocimiento, que ha incorporado nuevos desarrollos tecnológicos, formas adecuadas de concepción del aprendizaje, la naturaleza constructiva de la creación del conocimiento y la utilización de espacios virtuales de interactividad que promueven la enseñanza y el aprendizaje.

Con base en lo anterior, este trabajo expone cómo las comunidades de práctica (convertidas ahora en comunidades virtuales de aprendizaje) se han enriquecido con las aportaciones de enfoques teóricos como el conectivismo y la inteligencia colectiva, el desarrollo de los ambientes virtuales de aprendizaje, los espacios personales de aprendizaje, el uso de aplicaciones de la Web 2.0 y los cursos masivos (MOOC).

A continuación se describe el concepto de comunidades de práctica y sus características generales y, posteriormente, se actualiza el concepto con base en la noción de comunidades virtuales de aprendizaje y se presentan algunos desarrollos tecnológicos y pedagógicos actuales que enriquecen la educación a distancia.
\end{abstract}

Palabras clave:

Comunidades virtuales de aprendizaje, Comunidades de práctica, Educación a distancia

\begin{abstract}
:
This paper addresses the issue of the transformation of communities of practice as a collective strategy of knowledge construction, which has incorporated new technological developments, innovative forms of learning, constructive nature of knowledge creation and the use of virtual spaces of interactivity that promote teaching and learning.

This paper describes how communities of practice (now converted in virtual learning communities) have been enriched by the contributions of theoretical approaches as connectivism and collective intelligence, development of virtual learning environments, personal learning spaces, use of Web 2.0 applications and Massive Open Online Courses (MOOC).

The concept of communities of practice and their general characteristics is described and updated the concept based on the notion of virtual learning communities and some current technological and educational developments that enhance, mainly, distance education.
\end{abstract}

\section{Keywords:}

Virtual learning communities, communities of practice, Distance Education 


\section{Comunidades de práctica}

\section{Definición de las comunidades de práctica}

Se reconoce al educador suizo Etienne Wenger como el principal impulsor de las comunidades de práctica, las cuales entendió como una forma actualizada de construir aprendizaje con base experiencias prácticas colectivas de un determinado grupo social.

Recurriendo a este autor, los académicos del Colegio de México, Vega y Quijano (2010), definen "La comunidad de práctica es conceptualizada como un espacio de aprendizaje, negociación, significado e identidad, en donde los sujetos que participan se otorgan mutuamente un sentido de pertenencia a la comunidad y una participación "legítima", que puede ser central o periférica (Wenger, 1998)" (p.93).

De la anterior cita se desprenden varios principios básicos de las comunidades de práctica: son espacios de interacción, está conformado por un grupo social que construye su identidad por medio de la actividad desempeñada, el sentido de lo realizado (aprendizaje) se negocia entre los integrantes del colectivo, la comunidad permanece por voluntad de sus miembros y existen diversos niveles de participación de acuerdo al conocimiento de la actividad (a groso modo, expertos y aprendices) que se manifiesta en intervenciones centrales o periféricas.

Ruiz de Velazco (2005) describe que las comunidades de práctica son redes sociales que generan una transferencia informal interna de su conocimiento práctico, pero que, al mismo tiempo, cuentan con una estructura formal que fortalece la identidad del grupo y genera aprendizaje por medio de la participación y un liderazgo de tipo compartido.

Es necesario establecer que las comunidades de práctica, como espacio de aprendizaje por medio de la actividad social, han existido desde que el hombre lleva a cabo acciones colectivas para tener dominio de una función específica. Los talleres gremiales (herrería, ebanistería, laudería, por ejemplo) se han establecido, desde tiempos remotos, como comunidades que aprenden haciendo y reflexionando sobre lo producido.

Los educadores comenzaron a buscar estrategias innovadoras de enseñanza en la formación profesional que mostraran resultados efectivos a largo plazo. Es entonces cuando, afirman Coto Chotto, Corrales y Mora (2008), llegan a interesarse por las comunidades de práctica.

Debe entenderse que la comunidad de práctica es una estrategia de aprendizaje social basada en la participación colectiva de un grupo que recrea su identidad en su actividad cotidiana. Sus componentes principales son:

“1) Significado: una manera de hablar de nuestra capacidad (cambiante), en el plano individual y colectivo, de experimentar nuestra vida y el mundo como algo significativo;

2) Práctica: una manera de hablar de los recursos históricos y sociales, los marcos de referencia y las perspectivas compartidas que pueden sustentar el compromiso mutuo en la acción; 
3) Comunidad: una manera de hablar de las configuraciones sociales donde la persecución de nuestras empresas se define como valiosa y nuestra participación es reconocible como competencia;

4) Identidad: una manera de hablar del cambio que produce el aprendizaje en quiénes somos y de cómo crea historias personales de devenir en el contexto de nuestras comunidades" (Wenger, 2010, p. 22).

El aprendizaje en estos espacios tiene, como se aprecia en lo argumentado hasta ahora, una amplia dimensión cultural en la que el impacto del conocimiento se da a nivel individual y colectivo. Los sujetos desarrollan su aprendizaje y posterior conocimiento participando en la comunidad de la que forma parte y el colectivo llega a reconocer sus competencias y dominios generados por la inteligencia colectiva o capital intelectual que proviene de sus miembros. En estas condiciones el conocimiento producido dentro de grupos que poseen una identidad social compartida, llega a ser muy provechoso ya que se fundamenta en el compromiso y la significatividad de la práctica.

\section{Objetivos y propósitos de las comunidades de práctica}

Al ser un espacio social que genera múltiples interacciones, las comunidades de práctica permiten alcanzar objetivos y propósitos diversos.

Wenger (2010) hace hincapié en la construcción de identidad social en los participantes de un grupo de aprendizaje. Para este autor el tema es muy relevante ya que la generación de identidad establece un compromiso con la actividad colectiva, lo que repercute en una aprendizaje más sólido. El autor suizo señala que este objetivo se logrará siempre y cuando la comunidad permita hacer visibles las trayectorias de adquisición del conocimiento, lo cual es relevante por dos razones: porque el sujeto reconoce la situación de dominio o de conocimientos en la que se encontraba en el pasado y lo que ha tenido que realizar para constituir su práctica actual, y porque al conocer su trayectorias se compromete con su práctica en el escenario de un futuro deseado.

La constitución de comunidades de práctica en el medio universitario permite romper con los modelos pedagógicos basados en la transmisión de información centrados en el docente, para establecer, de acuerdo con Coto Chotto, Corrales y Mora (2008), una cultura académica de intercambio de conocimiento y experiencias entre profesores, lo que transforma la práctica docente al instituir el aprendizaje colaborativo como una estrategia pedagógica institucional.

Fernández y Oliveira (2009) apuntan a la utilidad que tienen las comunidades de práctica para resolver problemas concretos que requiere la participación de expertos, ya que el contar con profesionales en el grupo, permite aprovechar las experiencias y prácticas compartidas a través de espacios virtuales que generan una comunicación eficaz. 


\section{Características de las comunidades de práctica}

Como todo colectivo humano, llámese grupo o equipo, las comunidades de práctica tienen una serie de principios de operación y de estructura, con menor o mayor grado de formalismo, dependiendo el caso.

Al revisar la bibliografía disponible sobre este tema se puede sostener que una comunidad de práctica es un grupo no institucional creado exprofeso con el objetivo de que sus miembros, quienes comparten intereses comunes por la actividad realizada, dispongan de un espacio para interactuar e intercambiar experiencias y competencias voluntariamente sobre los temas que los congregan. Es un colectivo integrado por sujetos que poseen distintos niveles de dominio, lo cuales crean aprendizaje y conocimiento.

Las características principales de las comunidades de práctica, de acuerdo con Vega y Quijano (2010) citando a Wenger, son los siguientes:

“a) la coexistencia de relaciones de armonía y conflicto; b) el interés de sus miembros para realizar actividades de manera conjunta; c) los flujos adecuados y ágiles de información y difusión de la innovación; d) la existencia de un ambiente adecuado que permite la discusión de problemas sin mayor preámbulo; e) la existencia de un código no escrito de puntos en común entre todos sus integrantes; f) la conciencia sobre las habilidades y conocimientos de todos los miembros que conforman la comunidad; g) la comunicación entre sus miembros, que se da mediante una jerga común y términos específicos; y h) el compartir un discurso común sobre la visión del mundo" (p.93).

La comunidad, como todo grupo, tiene un ciclo de existencia, pero puede permanecer vigente siempre y cuando sus integrantes participen activamente y la maduración del colectivo les permita integrar temas actuales y novedosos que representen retos intelectuales, motivacionales y profesionales para que sus participantes continúen aprendiendo.

Los principios que sostienen a las comunidades de práctica, de acuerdo con Sanz (2005), son los siguientes:

a) Compromiso mutuo. Lo que se constituye como la principal ventaja de la comunidad de práctica, ya que se reconoce que la participación de cada uno de sus miembros es necesaria para construir aprendizaje. Se parte del principio que todos piensan más que uno.

b) Empresa conjunta. Los integrantes del grupo pueden tener interpretaciones diferentes de los objetivos por los que se crea la comunidad, pero comparten la misión de trabajar conjuntamente para llevar a cabo una actividad común que satisfaga a todos.

c) Repertorio compartido. Al madurar la comunidad, por medio de su práctica cotidiana, se va creando una cultura común que fortalece una identidad común, lo cual se observa en un lenguaje particular, actividades comunes, símbolos propios, dominio tecnológico similar, etc. 
Los principios arriba señalados requieren de una infraestructura de participación que debe incluir medios interactivos presenciales o virtuales, actividades a realizar de manera conjunta, apertura a la participación de nuevos miembros, logro de metas que puedan generar compromiso de los integrantes con la comunidad, tolerancia a la emisión de juicios y evaluaciones, utilización de instrumentos que desarrollen competencia y creación de una memoria de la comunidad en forma de documentación disponible.

Una característica fundamental de las comunidades de práctica, afirman Amorocho, Gómez y Andrade (2010) y que determina su posterior éxito, es el principio de que este colectivo debe gestionarse y administrarse para que las interacciones sociales se conviertan en relaciones de confianza, con objetivos concretos que alienten la creación de conocimiento.

\section{Roles en las comunidades de práctica}

Como ya se hizo mención, en las comunidades de práctica existen diversos grados de experiencia y conocimientos entre sus integrantes, lo cual es considerado una ventaja ya que en la estructuración del conocimiento se retoman varios referentes, por lo que, desde un punto de vista estructural, se generan varios roles.

Uno de ellos es el ser participante central o periférico. El primero es desplegado por aquellos miembros que intervienen estableciendo discusiones, generando propuestas de solución, retroalimentando activamente a sus compañeros. El segundo tipo de participante es aquel que representan los miembros de recién ingreso y que limitan su participación a leer contribuciones y solicitar resolución de dudas. Ambos papeles son determinantes para el crecimiento intelectual de la comunidad.

Otro rol clave lo representa el coordinador o moderador de la comunidad, quien tiene como propósito asegurar las condiciones para que el colectivo de aprendizaje desempeñe su práctica y permanezca en el tiempo. Sus principales actividades consisten en identificar temas relevantes, planificar las actividades del grupo, hacer interactuar a los participantes, procurar el crecimiento intelectual de los miembros, retroalimentar a los integrantes, resumir conclusiones y evidenciar el conocimiento adquirido.

Los principales roles en las comunidades de práctica, apuntan Vega y Quijano (2010), son:

a) Líderes. Son los que gestionan las actividades del grupo y proporcionan el apoyo necesario para su operación y sostenimiento desde un punto de vista técnico y motivacional.

b) Miembros experimentados. Son los expertos de la comunidad cuyas opiniones y contribuciones son consideradas confiables. Actúan como consultores y representan la memoria de las actividades de la comunidad de práctica.

c) Miembros regulares. Son aquellos que tienen participación activa en el grupo y que generan soluciones de forma colaborativa. 
d) Miembros principiantes. Son los integrantes que están experimentando una curva de aprendizaje y asimilación cultural que les permita participar de manera eficaz dentro de la comunidad.

e) Visitantes. Son observadores interesados en la actividad de la comunidad de práctica.

En el ámbito meramente educativo, los perfiles y papeles del profesor y del estudiante se modifican dentro de una comunidad de práctica. Los maestros pasan de ser reproductores de conocimiento a animadores, facilitadores y orientadores dentro del proceso de enseñanza y aprendizaje, lo que enriquece la función docente y su calidad. Los estudiantes centran su aprendizaje en ellos mismos a partir de la interacción grupal, toda vez que la comunidad se convierte en una fuente principal de información, interacción y motivación para generar conocimiento. Los estudiantes se hacen corresponsables del aprendizaje de todos.

\section{Tipos de comunidades de práctica}

Aunque todas parten del mismo principio de generación de aprendizaje y aplicación del mismo, existen diversos tipos de comunidades de práctica que varían de acuerdo a su ámbito, tamaño, propósitos, formas de interactuar, integración por perfiles de competencia, entre algunas categorías.

Por tipo de propósito se puede hablar de comunidades de interés (cuya actividad se enfoca al intercambio de información entre sus miembros sobre tópicos que los vinculan), comunidades de compromiso (se crean para cumplir con un proyecto o tarea específica, tienen una orientación más pragmática y menos reflexiva, y permanecerá mientras existan actividades que le den continuidad) y las comunidades de práctica propiamente dichas (son informales, voluntarias, se crean por interés común, son reflexivas y pragmáticas y constituyen los espacios ideales para el aprendizaje social).

Por la forma de interactuar se puede establecer que existen las comunidades presenciales (las que siempre interactúan cara a cara) las comunidades virtuales (las que solo operan utilizando una plataforma tecnológica que funciona conectada a internet) y las comunidades mixtas (que tienen tanto actividades presenciales como tareas que se realizan a distancia).

\section{Comunidades de práctica y Tecnologías de la Información y la Comunicación (TIC)}

Es un hecho que el desarrollo de las TIC ha establecido nuevas formas de interacción social, lo cual tiene efectos inmediatos en el ámbito educativo, que van más allá de aplicaciones didácticas diseñadas específicamente para mejorar el proceso de enseñanza-aprendizaje. En la actualidad se elaboran, como se verá más adelante, plataformas virtuales que buscan integrar, en espacios con diferentes grados de flexibilidad, herramientas que permitan aprovechar la inteligencia colectiva de los participantes en un grupo. El incesante desarrollo de las tecnologías está creando una 
diversificación de comunidades amplias y complejas que establecen nuevas economías de significado.

Es menester, entonces que los docentes aprovechen las posibilidades que se les presentan con el avance tecnológico ya que les permite establecer ambientes innovadores de aprendizaje, mejorar las comunicaciones sociales, interactuar activamente y utilizar el aprendizaje colaborativo en sus clases. El desarrollo tecnológico genera comunicaciones más libres, abiertas y democráticas con discursos son más directos que permiten mejorar los niveles de interactividad en nuevas formas de comunicación social. La horizontalidad en la comunicación borra las jerarquías, por lo que los participantes ganan confianza, mejoran sus reflexiones y se muestran más creativos y libres.

Al referirse a las ventajas que las TIC proporcionan a las comunidades de práctica, Sanz (2010) menciona:

"1. Visibilidad del experto de cara a la comunidad de práctica. Probablemente por las veces que interviene (números de mensajes) o por los comentarios que hacen el resto de los miembros sobre él, es mucho más fácil en un tipo de comunidades de práctica que utiliza como canal de comunicación las nuevas tecnologías identificar quién es el experto de la comunidad de práctica.

2. Mantener la memoria, por ejemplo, en temas de movilidad. El espacio de trabajo virtual común permite almacenar, organizar y descargar presentaciones, herramientas y otros materiales. Además, el sistema derepositorio y los metadatos permiten la identificación del autor del documento y facilitan la identificación del autor del documento, al mismo tiempo que el contexto en que se desarrolló, y refuerza la credibilidad y el valor del contenido.

3. Visibilidad de la comunidad de práctica. Permite entender el contexto a los nuevos incorporados. Con un simple vistazo, revisando los mensajes de la comunidad de práctica, un recién llegado puede captar y entender en qué consiste la actividad de la comunidad de práctica.

4. Relatos estructurados para preservar la memoria de la comunidad de práctica. Son relatos orales y entrevistas recogidas a través de tecnologías multimedia como audio o vídeo. En este apartado, los autores, haciendo un guiño al futuro, piensan en la posibilidad de dejar registros de conversacionesdonde conservar el vocabulario (palabras, conceptos o símbolos) de cara a nuevos miembros (repertorio compartido de Wenger)" (pp. 27-28).

Es evidente que el desarrollo de las TIC ha impactado en las comunidades de práctica quienes han ampliado su perfil original para aprovechar el desarrollo tecnológico en el ámbito de las redes sociales, las aplicaciones de la Web 2.0 y el aprendizaje móvil, entre otros. Se habla ahora de las comunidades virtuales de aprendizaje, tema que a continuación se desarrolla: 


\section{Comunidades Virtuales de Aprendizaje}

\section{Conceptos teóricos fundamentales para las comunidades virtuales de aprendizaje: inteligencia colectiva y conectivismo}

Son muchas las teorías y variadas las nociones que abonan a la construcción del concepto de comunidades virtuales de aprendizaje; sin embargo hay dos que sobresalen ya que se orientan a comprender la naturaleza de las redes sociales mediadas tecnológicamente y el papel principal que juega el capital intelectual en la creación colectiva de aprendizaje y conocimiento.

Al hacer referencia a la teoría de la actividad histórica cultural, inspirada en los planteamientos de Vygotsky, Miranda y Tirado (2012) mencionan que las prácticas sociales se llevan a cabo por actividades en las que el conocimiento y las acciones se distribuyen entre los miembros de la comunidad, así como en sus artefactos culturales en un contexto cultural determinado. Estas ideas presuponen que para llevar a cabo una determinada actividad social de aprendizaje es necesario considerar el conocimiento distribuido y las herramientas con las que cuentan cada una de las personas integrantes de una determinada comunidad cultural.

De acuerdo con Lévy (2004),la inteligencia colectiva es una inteligencia repartida dentro de una comunidad y valorada socialmente. Existe un escenario colectivo, al que el autor mencionado denomina espacio del conocimiento o inteligencia colectiva en el que se prepondera el reconocimiento y enriquecimiento mutuo de las personas, con la conciencia de que nadie lo sabe todo, por lo que se genera una efectiva movilización de competencias.

Esta noción resulta fundamental para la constitución de las comunidades virtuales de aprendizaje ya que toda construcción colectiva de conocimiento parte de la búsqueda e integración de las aportaciones intelectuales de sus miembros.

La inteligencia colectiva es la capacidad que posee un grupo para tomar decisiones sobre su propio futuro y para alcanzar sus objetivos, en un contexto complejo. Cobo (2007a) sostiene, siguiendo a Lévy, que es un capital no individual sino colectivo y que, como en una sociedad anónima, cada inversor aporta sus conocimientos, experiencia y actividad, con lo que la comunidad alcanza un nivel de inteligencia superior al intelecto individual contribuyente. Finalmente el autor menciona cuatro condiciones fundamentales para alcanzar una inteligencia colectiva: variabilidad en las opiniones de los miembros de un grupo, independencia de criterio, existencia de subgrupos en el colectivo y procedimientos para incorporar los juicios individuales en las decisiones colectivas.

Otra noción central para explicar la evolución de las comunidades de práctica, es la del conectivismo, teoría desarrollada por George Siemens y Stephen Downes, la cual explica el aprendizaje en la era digital con base en las redes de conocimiento existentes.

Los principios del conectivismo son, de acuerdo a Rodríguez y López (2013):

"1. El aprendizaje y el conocimiento se basan en la diversidad de opiniones. 
2. El aprendizaje es un proceso de conectar nodos especializados o fuentes de información.

3. El aprendizaje puede residir en dispositivos no humanos.

4. La capacidad de aprender es más importante que lo que se conoce hoy en día.

5. Es necesario cultivar y mantener conexiones para facilitar el aprendizaje continuo.

6. La capacidad de ver conexiones entre campos, ideas y conceptos es una capacidad fundamental.

7. La actualización del conocimiento es la intención de todas las actividades de aprendizaje conectivista.

8. La toma de decisiones es en sí un proceso de aprendizaje” (p. 415)

Es importante resaltar que son precisamente las redes sociales, como las comunidades virtuales de aprendizaje, las que generan conocimiento nuevo por medio de la identificación de las características que ofrecen los diferentes nodos que integran la red. El aprendizaje se produce cuando se ha determinado cuándo y dónde establecer contacto para obtener información relevante que produce conocimiento para la red en su conjunto.

De lo anteriormente expuesto se puede afirmar que el modelo de aprendizaje del conectivismo se ajusta a la sociedad del conocimiento ya que aprovecha las posibilidades colaborativas de las aplicaciones de la Web 2.0, el intercambio permanente de información, la existencia de comunidades de práctica y la cultura sociotecnológica de los llamados nativos digitales, quienes poseen una cultura tecnológica que los hace ser consumidores de información y creadores de contenidos por medio redes sociales, wikis y blogs.

\section{Definición de las comunidades virtuales de aprendizaje}

Probablemente la definición más completa para entender la comunidad virtual de aprendizaje es la proporcionada por Coll, Bustos y Engel (2007) presentada en la siguiente cita: "Desde nuestra perspectiva, una comunidad virtual es un grupo de personas o instituciones que se conectan a través de la red compartiendo el espacio virtual que han creado para tal fin (Coll, 2004). Para nosotros, lo fundamental de una comunidad virtual es reconocer que, gracias al desarrollo de las tecnologías de la información y la comunicación (TIC), surgen dinámicas específicas que potencian la aparición de nuevas formas de relación y de organización social y comunitaria” (p. 89).

Estos autores hacen hincapié en que el desarrollo tecnológico determina una forma distinta de establecer relaciones sociales mediadas por un espacio virtual que permite a las comunidades de aprendizaje funcionar como una red. Sostienen, también, que las comunidades virtuales están orientadas a tareas y objetivos preestablecidos y que el espacio tecnológicamente constituido, incrementa el vínculo grupal y potencializa el aprendizaje colectivo.

Las comunidades de aprendizaje, señalan Tirado y Martínez (2010), son grupos cuyos integrantes, por medio de una cultura compartida, se comprometen en un proceso de construcción de conocimiento colectivo. La horizontalidad de las relaciones que permite el cuerpo de pares, hace desaparecer las jerarquías, lo que potencia el desarrollo de la colectividad por medio de la expansión del conocimiento. Pineda, Meneses y Téllez (2013) sostienen que las comunidades virtuales deben verse como redes sociales que son mediadoras en el proceso de aprendizaje y que brindando la gestión pedagógica 
adecuada fomentan la comunicación permanente y efectiva, aprovechando la inteligencia colectiva del grupo para construir conocimiento utilizando tecnología.

Finalmente, Murua, Cacheiro y Gallego (2014) actualizan el concepto y definen como cibercomunidad de aprendizaje (más cercano al concepto de comunidad de práctica que al de comunidad de aprendizaje) al "grupo de personas (profesionales, estudiantes, gentes con intereses comunes...) comunicadas / vinculadas a través de redes / Internet de forma continuada para compartir información, ideas, reflexiones, experiencias... con el interés común de velar por su desarrollo personal, académico y profesional y el de los demás miembros que la componen" (p.21).

\section{Tipos de aprendizaje abordados por las comunidades virtuales}

Gea y colaboradores (2013) afirman que existen los siguientes tipos de comunidades virtuales de aprendizaje, diferenciados por la metodología didáctica utilizada:

a) Aprendizaje basado en prácticas. Se integra por un grupo de aprendices que está enfocado a conocer métodos para resolver problemas concretos en situaciones reales.Lo central es dotar a los miembros de estas comunidades de habilidades prácticas específicas.

b) Aprendizaje basado en conocimiento. Las personas que componen estas comunidades buscan ampliar, por medio del trabajo colectivo, la comprensión de las temáticas abordadas. Se orientan principalmente a identificar conocimiento nuevo para posteriores fines prácticos.

c) Aprendizaje basado en problemas. Es aquella que está integrada, en muchas ocasiones, por sujetos con formaciones diversas y que en un tiempo determinado, deben resolver de forma colaborativa un asunto que les fue asignado.

Cobo (2007b), amplia los tipos de aprendizaje que conforman a las comunidades de práctica, incluyendo los siguientes:

a) Aprender haciendo. Está basada en la utilización, por parte de los involucrados en el proceso de enseñanza-aprendizaje de diversas herramientas de la red para realizar proyectos colectivos, los cuales son supervisados y retroalimentados para generar un aprendizaje constructivista.

b) Aprender interactuando. Se logra mediante la utilización de diversas herramientas de interacción, como los foros, chats y comunicaciones de audio y video, por medio de los cuales los participantes aprenden unos de otros.

c) Aprender buscando. Es un proceso fundamental en la generación de conocimientos mediados tecnológicamente y se hace necesario cuando los estudiantes requieren realizar investigaciones y proyectos.

d) Aprender compartiendo: Para poder construir aprendizaje de forma colaborativa es indispensable compartir el conocimiento que tiene cada uno de los miembros de una comunidad de aprendizaje. Para tal fin se deben utilizar las diferentes aplicaciones, sitios y herramientas que están disponibles en la red, como los espacios para subir trabajos, presentaciones, podcast y videos, o el desarrollo de ambientes personales de aprendizaje. 


\section{Comunidades virtuales de aprendizaje y Tecnologías de la Información y la Comunicación (TIC)}

Como ya se ha señalado, el incesante desarrollo de las TIC ha impactado dramáticamente en el campo educativo de manera tal que, planeándolo o no, alumnos y profesores están incorporando la utilización de herramientas de la Web 2.0 para incrementar individual o socialmente su aprendizaje. Las comunidades de práctica se han enriquecido con la existencia de plataformas o ambientes virtuales de aprendizaje. Pineda y Meneses (2010) mencionan que las transformaciones generadas por el avance tecnológico están creando nuevos contextos sociales de comunicación basados en las redes sociales y en comunidades de aprendizaje que se constituyen como forjadoras de procesos educativos y sociales. La creación de comunidades online desarrolla un sentimiento de pertenencia comunitario que se consolida por la interacción y la comunicación sociales.

Las TIC han permitido no solo establecer redes sociales y diversas comunidades que buscan generar conocimiento nuevo, sino, también, como manifiestan Padilla y López de la Madrid (2013) crear materiales educativos enriquecidos y, por medio del diálogo didáctico intersubjetivo entre profesor y alumno, alentar aprendizajes basados en situaciones que problematicen lo complejo del mundo real.

\section{Características de las comunidades virtuales de aprendizaje}

Las comunidades virtuales de aprendizaje tienen como característica principal, afirman Coll, Bustos y Engel (2007), el incentivar la participación y comunicación entre sus miembros mediante la flexibilidad del entorno, lo que incrementa el convencimiento de que la construcción de conocimiento se genera por la interacción social y la elaboración conjunta de significados. Por su parte, Tirado y Martínez (2010), identifican los siguientes rasgos definitorios de las comunidades virtuales: sentimiento de comunidad, expectativas comunes de aprendizaje, condiciones de apoyo y confianza, cooperación e interacción, participación respetuosa, discurso progresivo a través de la construcción de conocimiento y apropiación mutua del aprendizaje.

Existen dos formas de entender a las comunidades virtuales de aprendizaje, según Cabero (2013): desde una aproximación tecnológica-instrumental y desde su característica pedagógica-educativa. La primera se refiere, específicamente, a las herramientas tecnológicas existentes, de la Web 2.0 y 3.0, que son utilizadas para ampliar el aprendizaje y generar conocimientos y, la segunda a los sistemas de organización y gestión que establecen la dinámica por medio de la cual los integrantes de la comunidad participan y reciben apoyo para la construcción de su propio aprendizaje y lograr la metas establecidas colectivamente. En lo que se refiere a lo tecnológico-instrumental, este autor señala a la interacción mediada por dispositivos móviles, la flexibilidad en cuanto al tiempo de interacción, la diversidad de ubicaciones geográficas, las posibilidades de comunicación sincrónica y asincrónica, la comunicación multimediática, las interacciones uno a uno o con todo el grupo y el objetivo compartido de construir conocimiento.

Ruiz de Velazco (2005) hace hincapié en la necesidad de que los participantes en una comunidad de aprendizaje cumplan con una serie de prerrequisitos necesarios para asegurar la buena marcha del mismo, tales como: 
a) Búsqueda de información. Uso de conceptos clave, búsqueda en bases de datos confiables, utilización de operadores booleanos, interpretación de resultados de búsqueda, herramientas de búsqueda avanzada, almacenamiento de fuentes encontradas, entre otras.

b) Clasificación, discriminación y selección de la información. Técnicas de clasificación y selección de la información encontrada.

c) Recuperación de información. Estrategias para buscar fuentes ya revisadas en diferentes bases de datos.

d) Uso de la información. Desarrollar técnicas para analizar, criticar y hacer reflexiones sobre el material recolectado. Realización de ensayos o técnicas de síntesis de las fuentes recuperadas.

e) Socialización de la información. Establecer espacios para compartir la información recopilada y análisis desarrollados para que estén disponibles, virtualmente, a los miembros de la comunidad.

f) Colaboración en proyectos. Saber trabajar de forma colaborativa con los miembros de la comunidad, lo que implica el saber compartir, ser responsable del propio aprendizaje y el de los demás, comunicarse de manera efectiva y comprometerse con los objetivos del grupo.

En cuanto a las características de las comunidades virtuales enfocadas al aprendizaje constructivista, Padilla y López de la Madrid (2013) mencionan las siguientes:

a) Crean un marco de cooperación didáctica. Toda vez que los participantes en las actividades educativas de la comunidad, negocian los significados del aprendizaje mediados por el docente, partiendo del hecho de que todos los integrantes del grupo parten de culturas diferentes con conocimientos previos diversos.

b) Crean espacios de interacción que permiten a los alumnos trabajar entre ellos de forma conjunta y con el profesor. Esto se logra al utilizar el espacio virtual e involucrando a los participantes en la actividad educativa, aprendiendo de sus propios compañeros.

c) Crean un equilibrio entre el material cultural que se pone a disposición del alumno para su propio aprendizaje y la guía y orientación del profesor. Lo anterior por medio de la participación permanente del profesor quien, en la educación a distancia, tiene como una de sus funciones principales apoyar a los estudiantes en la construcción de su propio conocimiento.

d) Aseguran el desarrollo de procesos de pensamiento complejo. Para lo cual se llevan a cabo actividades fundamentadas en técnicas didácticas como el aprendizaje basado en proyectos y el estudio de casos las cuales están orientadas a que el estudiante elabore estrategias de solución de problemas, incorporando su experiencia previa.

e) Favorecen el desarrollo de una interacción orientada hacia la creación de zonas de desarrollo próximo. Lo que requiere que el profesor genere apoyos específicos de acuerdo a las necesidades de los alumnos para que puedan pasar de un nivel de conocimiento inferior a uno cualitativamente más complejo y, por ende, superior. Con estas intervenciones, el profesor hace posible que la comunidad virtual sea un espacio de aprendizaje al incentivar el desarrollo individual y colectivo de conocimientos nuevos. 
f) Incluyen tareas auténticas de aprendizaje que permiten diversificar los estilos de aprendizaje de los alumnos. Con base en suprimir contenidos textuales, para crear actividades contextualizadas a la realidad de los alumnos.

\section{Objetivos de las comunidades virtuales de aprendizaje}

Después de haber revisado las principales características de estructura, función y tipos de aprendizaje que persigue, se presentan los propósitos y objetivos que se intentan alcanzar al establecer comunidades virtuales.

El objetivo central de las comunidades virtuales de aprendizaje es, de acuerdo con Miranda y Tirado (2012), desarrollar el pensamiento profundo mediante la participación proactiva a través de acciones colaborativas como la escritura en grupo, el cual es una acto creativo. Mencionan la importancia de contar con medios asincrónicos de comunicación, los cuales permiten que los participantes dispongan de tiempo suficiente para reflexionar e investigar, lo que genera un pensamiento crítico. En la misma línea argumentan Padilla y López de la Madrid (2013) ya que indican que los entornos virtuales provocan la aparición de procesos de tipo intra e intersubjetivos. Intrasubjetivos ya que los miembros de la comunidad internalizan, mediante la construcción social del conocimiento, nuevos contenidos que modifican su estructura cognitiva e intersubjetivos, toda vez que la interactividad de los miembros constituye una red que logra puntos de concordancia en un proceso educativo colaborativo.

El objetivo de las comunidades virtuales consideran Pineda y Meneses (2013) es, dentro de la universidad en una sociedad digital, aprovechar la inteligencia colectiva utilizando la tecnología disponible para crear sujetos competentes en la sociedad del conocimiento, con sólidas capacidades humanas, cognitivas y técnicas. Por su parte, Cabero (2013) observa que las comunidades de práctica responden a la necesidad que tienen las sociedades de conocimiento actuales de generar aprendizajes permanentes en diversos contextos de formación, tanto de tipo formal como no formal e informal.

Para el ámbito escolar, la comunidad virtual de aprendizaje supone una estrategia de construcción colectiva de conocimiento que vincula sistemas pedagógicos actuales basados en el aprendizaje colaborativo, la construcción social del conocimiento y el desarrollo de la inteligencia colectiva, aprovechándose de la evolución de las TIC que ayudan a construir espacios virtuales de colaboración. A este respecto, González, García y Gonzalo (2011) expresan que el mayor potencial se halla en los alumnos, ya que la creación de sitios de interacción como los edublogs favorecen el aprendizaje colaborativo y autónomo.

\section{Diseño de comunidades virtuales de aprendizaje}

Como se desprende de la argumentación hasta ahora expuesta, hay condiciones tecnológicas, sociales y pedagógica-didácticas para establecer comunidades virtuales de aprendizaje en escenarios institucionales y no institucionales, que fomenten un aprendizaje expansivo y para toda la vida. Sin embargo es menester considerar ciertos elementos de diseño. 
Zúñiga y Arnáez (2010) mencionan las siguientes: valoración de la disponibilidad tecnológica y de la conectividad, conocer a los participantes y sus expectativas, determinar con claridad el papel que funge el facilitador, seleccionar el material de aprendizaje que constituye la mediación tecnológica y elegir el espacio virtual de interacción y las herramientas a utilizar. Estos mismos autores proponen reconocer cinco etapas para gestionar comunidades virtuales exitosas:

1) "Motivación: útil para reconocer la conveniencia de participar en una CVA para satisfacer una necesidad sentida.

2) Socialización: comprende el establecimiento de identidades virtuales y relaciones entre las personas participantes.

3) Intercambio: conlleva conseguir y compartir la información relevante a las necesidades propias, de otros y de la comunidad. Valora la eficiencia y los beneficios del nuevo proceso.

4) Construcción: se refiere al logro de la construcción coparticipativa de nuevos conocimientos y realidades.

5) Trascendencia: integra el proceso con otras formas de aprendizaje, extiende y multiplica la comunidad, creando una red de redes. El participante utiliza el proceso para lograr metas personales" (pp.24-25)

Finalmente, Cabero (2013) agrega otras condiciones a la lista: asegurarse de que todos los miembros de la comunidad puedan participar, contar con tecnologías amigables y de fácil acceso, dominio tecnológico básico, reglas claras para el trabajo colaborativo, gestión dinámica del grupo y promoción de la autorregulación de los estudiantes.

\section{Roles del docente y los alumnos en las comunidades virtuales de aprendizaje}

En otro apartado de este documento se hizo mención que las redes sociales y las formas de comunicación directa que permiten las aplicaciones de la web y las plataformas virtuales de aprendizaje, generan horizontalidad en las relaciones sociales dentro de una comunidad virtual, lo cual debe verse como una virtud, toda vez que produce autonomía y creatividad y empodera al grupo en la construcción de su propio conocimiento. Sin embargo es importante que tanto profesores como alumnos asuman el rol que les corresponde en estos colectivos de aprendizaje.

Padilla y López (2013) identifican las acciones que debe realizar un profesor dentro de una comunidad virtual de aprendizaje, para obtener los resultados deseados:

a) "acciones para organizar y gestionar la actividad en el aula virtual

b) acciones para orientar y dar sentido al aprendizaje

c) acciones de apoyo al clima socioemocional del aula

d) acciones para la exploración de los conocimientos previos de los estudiantes

e) acciones al servicio de la elaboración de representaciones cada vez más complejas y expertas de contenidos de enseñanza aprendizaje" (p.111).

Los alumnos requieren prepararse para generar procesos de razonamiento crítico por medio del cuestionamiento, en lugar de un razonamiento memorístico y repetitivo. Requieren generar autorregulación como condición necesaria para que puedan participar 
activa y provechosamente en la comunidad de aprendizaje. La autorregulación se basa en los siguientes aspectos: establecimiento de objetivos, planificación de actividades, automotivación, atención plena, estrategias de aprendizaje, autocontrol, autoevaluación y autorreflexión.

\section{Comunidades virtuales de aprendizaje y desarrollo de plataformas de interactividad}

A continuación se abordarán las diversas herramientas tecnológicas que se constituyen como aulas virtuales y que posibilitan, en grados diversos, el desarrollo de las comunidades virtuales de aprendizaje. Se describirán los entornos virtuales de aprendizaje (EVA) y los entornos personales de aprendizaje (EPL).

Al considerar el nivel alcanzado por las herramientas de interactividad en la red, las aplicaciones de la Web 2.0, las plataformas virtuales y las redes sociales se puede establecer una alianza de integración tecnológica-pedagógica que beneficie el proceso educativo, al estar en condiciones de crear espacios para que los alumnos creen, desarrollen y compartan contenidos e ideas.

Una valiosa herramienta para generar interacción entre los estudiantes para la construcción de conocimiento son los llamados entornos virtuales de aprendizaje o entornos virtuales de enseñanza-aprendizaje, los cuales son aplicaciones informáticas que facilitan la interacción entre los participantes, ordenan los contenidos de aprendizaje y muestran las actividades a realizar. Son indispensables en los modelos de educación a distancia, auxiliares en los mixtos y muy útiles en los presenciales.

Los profesores que administran los EVA deben orientarse a promover el aprendizaje permanente, por medio de su flexibilidad y orientación a la innovación; alentar comunicaciones en línea, establecer contenidos apropiados, cohesionar a la comunidad virtual de aprendizaje, tener dominio de la materia a impartir y de la plataforma a utilizar. Los EVA, de acuerdo con Silva y Romero (2014), hacen posible transitar de modelos pedagógicos tradicionales trasmisivos a uno orientado a la construcción compartida de conocimientos en la que los estudiantes se convierten en agentes de su propio aprendizaje y los profesores en facilitadores del proceso constructivo de los alumnos.

Pero para lograr que los estudiantes logren aprendizajes colaborativos, autónomos, autodirigidos y autorregulados, señalan Ampudia y Trinidad (2012), es necesario que los EVA no sean rígidos, burocratizados, centralizados y complicados. Que no se presenten como un reto a descifrar y que, al contrario, promuevan la participación libre.

Para que los docentes tengan un dominio del EVA y se aproveche la plataforma de aprendizaje, se requiere que participen en programas de formación pedagógica como el constructivismo, de habilidades tecnológicas, de diseño de actividades de aprendizaje y de gestión apropiada del entorno virtual.

Finalmente, Rodríguez y López (2013) señalan que diversos estudios sobre el aprendizaje mediado tecnológicamente son enfáticos al proponer evitar los EVA como simple repositorio de material educativo, por lo que se deben emplear herramientas de comunicación y redes personales de aprendizaje. 
Buscando mayor flexibilidad y autonomía en el proceso educativo, se crean los entornos personales de aprendizaje, los cuales están orientados, principalmente, al desarrollo del conocimiento del alumno. Ampudia y Trinidad (2012) describen las principales características de los EPL: los estudiantes los crean para generar el conocimiento que necesitan por medio de las herramientas disponibles en la Web 2.0; tiene una estructura flexible, abierta y autónoma; tiene como filosofía el compartir de abajo hacia arriba y es auto organizada y centrada en el alumno y el docente. Aunque, señalan, no hay una definición del concepto, se le reconoce como una metodología o conjunto de herramientas para el aprendizaje, que buscan organizar y compartir fuentes de información y conocimientos dentro de la red.

La diferencia principal entre los EVA y los EPL es que los primeros están diseñados de forma institucional, utilizando plataformas que varían en su grado de rigidez, mientras que los segundos se establecen de manera autónoma utilizando aplicaciones libres para compartir información. Rodríguez y López (2013) coinciden en que las nuevas aplicaciones de interacción de la web 2.0 es la que genera las diferencias ya que en el EPL se hace énfasis en la dimensión social y personal del aprendizaje y en los EVA en el valor de la tecnología de las plataformas de enseñanza.

Aunque los conceptos aquí abordados están plenamente diferenciados y que, al parecer, se encuentran a la alza los entornos personales de aprendizaje en detrimento de los ambientes virtuales de aprendizaje rígidos, lo que debe considerarse es que si se diseñan ambientes virtuales de aprendizaje autogestionados, autónomos y flexibles se pueden encontrar coincidencias con los EPL que faciliten el acceso a aplicaciones educativas abiertas y a las redes sociales de libre acceso.

Para concluir con este apartado, solo se hará mención de otra forma de gestionar contenidos para comunidades virtuales de aprendizaje que está en plena expansión, es decir los cursos online masivos y abiertos (MOOC, por sus siglas en inglés) los cuales de acuerdo a Gea y colaboradores (2013) "se basan en un modelo de aprendizaje social, fomentando la creación de verdaderas comunidades de aprendizaje. La tecnología usada está basada en Elgg, una plataforma OpenSource para la creación de comunidades" (p. 464)".

Los MOCC pueden atender a comunidades virtuales de aprendizaje compuestas por millones de estudiantes provenientes de diversos países del mundo que se interesan, como en las comunidades de práctica, en generar conocimientos específicos apropiados para sus actividades cotidianas profesionales o no profesionales orientadas a la adquisición de un conocimiento práctico. Estas comunidades virtuales son guiadas por un modelo de aprendizaje para toda la vida enfocado a procesos o saberes específicos, que difícilmente se abordan en la formación académica actual y que representan la adquisición de habilidades necesarias para los puestos de trabajo actuales.

\section{Reflexión final}

El presente documento representa una revisión retrospectiva de la noción de comunidades de práctica, recuperando de ella la característica principal de ser un espacio colectivo y libre de construcción de conocimientos y aprendizaje. Con el 
desarrollo de las nuevas tecnologías, de perspectivas pedagógicas fundamentadas en ella y la comprensión de su impacto social en usuarios con competencias y habilidades novedosas, se da pie a su enriquecimiento en comunidades virtuales de aprendizaje.

Para culminar el escrito que se refiere a aspectos tecnológicos y pedagógicos, se presenta la importancia de la plataforma de aprendizaje, la cual apoya la educación a distancia potenciando la inteligencia colectiva y el aprendizaje en red.

Profesores y alumnos tienen ante sí la oportunidad de potenciar su aprendizaje, expandir su conocimiento por medio de la interacción en espacios creados para ese fin y constituir auténticas sociedades del conocimiento.

Presentación del artículo: 20 de septiembre de 2015

Fecha de aprobación: 27 de septiembre de 2015

Fecha de publicación: 30 de octubre de 2015

González-Pérez, M.A. (2015). Enriquecimiento tecnológico y psicopedagógico del concepto de comunidades de práctica en la educación a distancia. RED. Revista de Educación a Distancia, 47(7). 30 de Octubre de 2015. Consultado el (dd/mm/aa) en http://www.um.es/ead/red/47

\section{Referencias}

Amorocho, Y., Gómez, L.C. y Andrade, H. (2010) De las redes sociales a las comunidades de práctica en el ámbito educativo. En Revista Educación en Ingeniería, jun, No.9, pp. 1-11.

Ampudia, V. y Trinidad, L. H. Entornos Personales de Aprendizaje: ¿final o futuro de los EVA?. En Reencuentro Universidad Autónoma Metropolitana Unidad Xochimilco, núm. 63, enero-abril, 2012, pp. 32-39

Cabero, J. (2013). El aprendizaje autorregulado como marco teórico para la aplicación educativa de las comunidades virtuales y los entornos personales de aprendizaje. Teoría de la Educación: Educación y Cultura en la Sociedad de la Información, 14 (2), 133-156.

Cobo, C. (2007a). Intercreatividad y Web 2.0. La construcción de un cerebro digital planetario. Cobo, C. y Pardo, H. (2007) Planeta Web, 2.0 Inteligencia Colectiva o Medios Fast Food: UVIC y FLACSO México: México.

Cobo, C. (2007b). Aprendizaje colaborativo: nuevos modelos para usos educativos. Cobo, C. y Pardo, H. (2007) Planeta Web, 2.0 Inteligencia Colectiva o Medios Fast Food: UVIC y FLACSO México: México. 
Coll, C., Bustos, A., \& Engel, A. (2007) Configuración y evolución de la comunidad virtual MIDE/DIPE: retos y dificultades. En Revista Electrónica Teoría de la Educación. Educación y Cultura en la Sociedad de la Información, vol. 8, No. 3, pp. 86-104.

Coto Chotto, M., Corrales, X. y Mora, S. (2008) Comunidades de práctica virtuales: Un enfoque para el desarrollo profesional docente. Ponencia presentada en el II Congreso Iberoamericano Virtual y Presencial. Educación y Sociedad del Conocimiento, Heredia, Costa Rica.

Fernández, P. y Oliveira, I. (2009) Horizontalidad discursiva en comunidades de práctica digitales. En CLAC Círculo de lingüística aplicada a la comunicación, v. 39 , pp. 35-55.

Gea, M. Et al (2013). Formación abierta sobre modelos de enseñanza masivos: nuevas tendencias hacia el aprendizaje social. En IV Congreso Internacional sobre Calidad y Accesibilidad de la Formación Virtual (pp. 460-467).

González, R., García, F. E., \& Gonzalo, N. (2011). Los edublogs como herramienta facilitadora en comunidades virtuales de aprendizaje. RELADA-Revista Electrónica de ADA-Madrid, 5 (3).

Lévy, P. (2004). Inteligencia colectiva. Por una antropología del ciberespacio.

Disponible en: http://www.minipimer.tv/txt/20110120/Inteligencia-Colectiva-PierreLevy.pdf

López, J.L. y Mata, G. (2012) Red social online como entorno virtual de aprendizaje. En Hospitalidad- ESDAI, julio-diciembre, pp. 95-113.

Miranda, A., y Tirado, F. (2012). Las nuevas universidades: El fenómeno de comunidades de aprendizaje en línea. En Revista de la educación superior, 41 (164), 9-33.

Murua. I., Cacheiro, M. L. y Gallego, D. (2014) Las cibercomunidades de aprendizaje (cCA) en la formación del profesorado en RED, Revista de Educación a Distancia, núm. 43, octubre-noviembre, pp. 52-80.

Padilla, S., \& López de la Madrid, M. C. (2013). Competencias pedagógicas y función docente en las comunidades virtuales de aprendizaje. Estudios pedagógicos (Valdivia), 39 (ESPECIAL), 103-119.

Pineda, E., Meneses, T. y Téllez, F. R. (2013). Análisis de redes sociales y comunidades virtuales de aprendizaje. Antecedentes y perspectivas. Revista Virtual Universidad Católica del Norte,1 (38), 40-55.

Rodríguez, M. R. y López, A. (2013). Entorno virtual de aprendizaje compartido en Educación Superior. En REDU Revista de Docencia Universitaria, 11 (1), 411-428. 
Ruiz Velasco, E. (2005). El aprendizaje colaborativo en entornos virtuales: comunidades que aprenden en comunidad. Ponencia presentada en el Virtual Educa 2005, Ciudad de México, México.

Sanz, S. (2005) Comunidades de práctica virtuales: acceso y uso de contenidos. En Revista de Universidad y Sociedad del Conocimiento, nov.vol. 2, No. 2, pp. 26-35.

Silva, J. E. y Romero, M. (2014). La Virtualidad una oportunidad para innovar en educación: un modelo para el diseño de entornos virtuales de aprendizaje. En Revista Didasc@lia: Didáctica y Educación, 5 (1), pp. 1-23.

Tirado, R. y Martínez, J. M. (2010). Creando comunidades virtuales de aprendizaje: análisis del progreso de las interacciones. En Revista de Educación, 353, 297-328.

Vega, M. y Quijano, A. (2010) Comunidades de práctica y alfabetización informacional. En IBERSID Revista de sistemas de información y comunicación, vol. 4, pp. 93-103.

Wenger, E. (2001). Comunidades de práctica: aprendizaje, significado e identidad. Barcelona: Paidós.

Zúñiga, C. y Arnáez, E. (2010). Comunidades virtuales de aprendizaje, espacios dinámicos para enfrentar el Siglo XXI. Tecnología en marcha, 23 (1), pp. 19-28 